\title{
Recurrent intracranial melanocytoma associated with a nevus of Ota
}

\author{
I. Rutten ${ }^{1}$, S. Bolle ${ }^{1}$, B. Kaschten ${ }^{2}$, A. Stevenaert ${ }^{2}$, J.-M. Deneufbourg ${ }^{1}$, and M. Deprez ${ }^{3}$ \\ ${ }^{1}$ Department of Radiotherapy, Centre Hospitalier Universitaire de Liège, Sart Tilman, Belgium \\ ${ }^{2}$ Department of Neurosurgery, Centre Hospitalier Universitaire de Liège, Sart Tilman, Belgium \\ ${ }^{3}$ Department of Pathology, Centre Hospitalier Universitaire de Liège, Sart Tilman, Belgium
}

\section{Introduction}

The nevus of Ota is a rare skin lesion which results from a neural crest disorder [3]. It is sometimes associated with meningeal melanocytic tumours [2].

Little is known about the therapeutic management of these lesions, once surgery has been performed. Indeed, the biological behaviour of these rare tumours has not been well characterized. Here we report the case of a patient with a relapsing intra-cranial melanocytoma showing an atypical pattern of brain infiltration at the time of recurrence.

\section{Case report}

This 37-year-old Caucasian female presents with complains of morning headaches having lasted for 6 months, loss of visual acuity together with mood disturbances. She describes episodes of vague malaise, possibly of comitial origin. On examination, the patient presents a large right-sided cutaneous grey-brown peri-orbital lesion which has been diagnosed as a congenital nevus of Ota involving the territory of the first branch of the right trigeminal nerve (Fig. 1a).

Neurological examination is normal. Ophthalmological examination shows an oculo-dermal melanosis and a left inferior lateral homonymous quadranopsia.

Bilateral carotid angiograms show a large lesion located on the right lateral wall of the supra-orbital region. Brain MRI demonstrates a $6 \mathrm{~cm}$ meningeal tumour at the olfactory groove and the adjacent part of the orbital ceiling, compressing the adjacent brain and shifting the midline structures to the left.

At the time of surgery (December 97), the incised skin and subcutaneous tissue show an abundant melanosis. This black-brown discolouration also extents to the dura and the skull base. The opening of the dura reveals a 6 $\mathrm{cm}$ tumour broadly located on the olfactory groove, with black pigmentation and haemorrhagic spots, well delineated from the adjacent brain parenchyma.

Microscopic examination shows a melanin-rich leptomeningeal tumour with a broad dural base. The tumour consists of uniform oval to spindle cells arranged in interlacing fascicules. The cells have elongated nuclei with finely dispersed chromatin and small nucleoli (Fig. 1b). Mitotic figures are rare, with less than 3 mitosis/50 high power fields (HPF) and there is no necrosis. Immunohistochemistry shows positive staining for protein S-100 and HMB-45, and negative staining for Vimentin, Glial Fibrillary Acidic Protein (GFAP) and Epithelial Membrane Antigen (EMA). The Ki-67 proliferative index is below 5\%. Ultrastructurally, the tumour cells have dendritic-like processes, few intermediate junctions and a large number of pre-melanosomes and melanosomes at different stages of differentiation. The cells do not exhibit the desmosomes and interdigitating cytoplasmic processes which are so characteristic of meningiomas. The ultrastructural and immunohistochemical features are therefore unequivocally in favour of a tumour of melanocytic origin. In the absence of other known malignancy, this clinically and histologically benign tumour is reported as a primary meningeal melanocytoma.

Post-operative MRI shows no residue. The patient receives no additional treatment and resumes a normal life. Control MRIs are performed yearly and remain negative for the following 4 years.

In 2002, a multifocal relapse is detected on the follow-up MRI in the absence of clinical signs or symptoms. 
Fig. 1. (a) Clinical aspect of the naevus of Ota, involving the skin of the right frontal and periorbital regions, as well as the upper conjunctiva. (b) light microscope photomicrographs of the tumour resected in December 1997 showing uniform melanin-rich spindle cells arranged in interlacing fascicules $(B 1 \times 200 ; B 2 \times 400)$. (c) light microscope photomicrographs of the largest nodule resected in July 2002. Brain invasion is obvious in the form of isolated HMB45 positive pigmented cells infiltrating the parenchyma and of perivascular cuffs of tumour cells distending the Virchow-Robin spaces $(C 1 \times 100 ; C 2$ and $C 3 \times 250)$. Chronic reactive astrocytic gliosis takes the form of granular eosinophilic bodies and Rosenthal fiberlike cytoplasmic enlargements $(C 4 \times 400)$

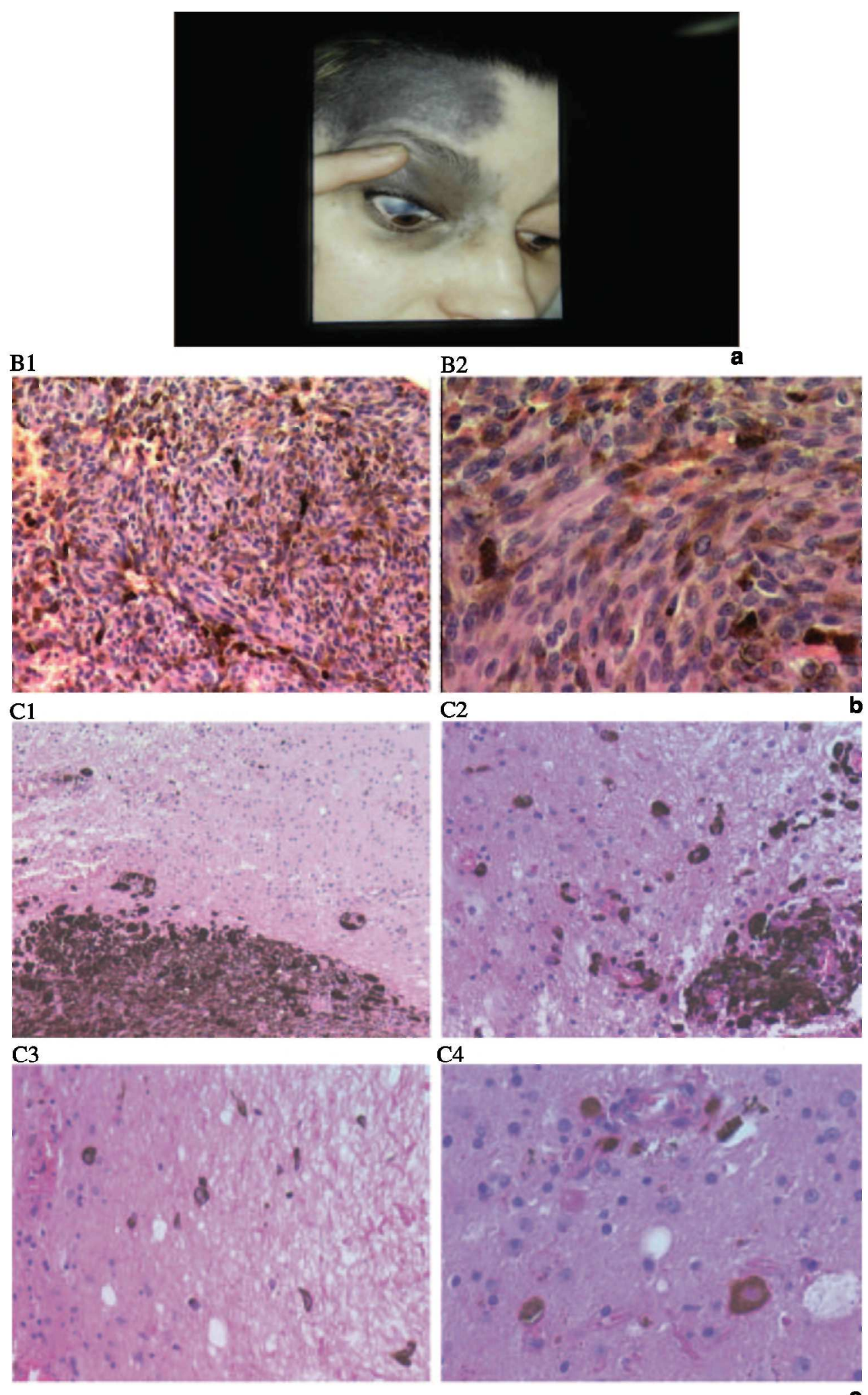


A second surgical resection is performed in July 2002 for a $3 \mathrm{~cm}$ nodule growing inside the previous operative cavity, and surrounded by 3 additional small nodules which lie bilaterally on the skull base and are embedded in a melanotic dura. Macroscopically, all these lesions have a similar blackberry like colour.

The histological aspect of the tumour recurrences (Fig. 1c) is similar to the former specimen resected in December 1997. There is no cytonuclear pleomorphism or intra-tumoural necrosis. Occasional mitotic figures are seen but do not exceed 1/10 HPF and the proliferative index is 5-6\% after nuclear labelling with Ki-67. However, the largest nodule shows obvious signs of brain invasion in the form of isolated HMB45 positive pigmented cells infiltrating the parenchyma and of perivascular cuffs of tumour cells distending the VirchowRobin spaces. The surrounding cortex and subcortical white matter show prominent chronic reactive astrocytic gliosis with the formation of granular eosinophilic bodies and Rosenthal fiber-like cytoplasmic enlargements. This atypical infiltrative pattern is interpreted by us as a sign of potential progression towards a more malignant phenotype.

Radiation therapy is administered on the volume of the initial extension at the dose of 54 Gy on the $95 \%$ isodose, given in fractions of $1.8 \mathrm{~Gy}$.

At the last control visit, 24 months later, there is no evidence of tumour residue or relapse on MRI. The patient is symptom free except for a mild loss of the sense of smell on the right side.

\title{
Discussion
}

Some authors conclude that even melanoma-like lesions associated with a nevus of Ota are not too aggressive [24]. However, others are more pessimistic, especially in Asian and Black patients [1, 5]. Therefore, in our opinion, the rather unpredictable evolution of this type of lesion warrants at least a close surveillance.

\section{References}

1. Arunkumar MJ, Ranjan A, Jacob M, Rajshekhar V (2001) Neurocutaneous melanosis: a case of primary intracranial melanoma with metastasis. Clin Oncol 13: 52-54

\author{
2. Balmaceda CM, Fetell MR, Powers J, O'Brien JL, Housepian EH (1993) Nevus of Ota and leptomeningeal melanocytic lesions. \\ Neurology 43: 381-386 \\ 3. Piercecchi-Marti M-D, Mohamed H, Liprandi A, Gambarelli D, Grisoli F, Pellissier J-F (2002) Intracranial meningeal melanocytoma \\ associated with ipsilateral nevus of Ota. J Neurosurg 96: 619-623
}

4. Rivers JK, Bhayana S, Martinka M (2001) Dural melanoma associated with ocular melanosis and multiple blue nevi. J Cutan Med Surg 5: 381-385

5. Theunissen P, Spincemaille G, Pannebakker M, Lambers J (1993) Meningeal melanoma associated with nevus of Ota: case report and review. Clin Neuropathol 12: 125-129

\section{Comment}

An interesting and well presented paper describing a recurrent melanocytic intracranial tumour in a patient with a nevus of Ota. The main focus of the paper is the perceived malignant transformation in the intracranial melanocytoma.

The authors have defined what they feel is progression to a malignant phase for this tumour - they have included convincing illustrations of brain invasion. Given that melanocytoma is generally considered to be a tumour with benign behaviour, the aggressive behaviour illustrated in this case is a good lesson for clinical neurosurgery.

\section{R. O. Wetter}

Southampton 